\title{
Extended Magnetic Exchange Interactions in the High-Temperature Ferromagnet MnBi
}

\author{
T.J. Williams, ${ }^{1,}$ a) A.E. Taylor, ${ }^{1}$ A.D. Christianson, ${ }^{1,2}$ S.E. Hahn, ${ }^{3}$ R.S. Fishman, ${ }^{4}$ D.S. Parker, ${ }^{4}$ M.A. McGuire, ${ }^{4}$ \\ B.C. Sales, ${ }^{4}$ and M.D. Lumsden ${ }^{1}$ \\ 1) Quantum Condensed Matter Division, Neutron Sciences Directorate, Oak Ridge National Lab, Oak Ridge, TN, \\ 37831, USA \\ ${ }^{2)}$ Department of Physics 83 Astronomy, University of Tennessee, Knoxville, TN, 37966, \\ USA \\ ${ }^{3)}$ Neutron Data Analysis $\&$ Visualization Division, Neutron Sciences Directorate, Oak Ridge National Lab, \\ Oak Ridge, TN, 37831, USA \\ ${ }^{4)}$ Materials Science and Technology Division, Physical Sciences Directorate, Oak Ridge National Lab, Oak Ridge, \\ TN, 37831, USA
}

(Dated: 11 September 2018)

The high-temperature ferromagnet $\mathrm{MnBi}$ continues to receive attention as a candidate to replace rare-earthcontaining permanent magnets in applications above room temperature. This is due to a high Curie temperature, large magnetic moments, and a coercivity that increases with temperature. The synthesis of MnBi also allows for crystals that are free of interstitial Mn, enabling more direct access to the key interactions underlying the physical properties of binary Mn-based ferromagnets. In this work, we use inelastic neutron scattering to measure the spin waves of $\mathrm{MnBi}$ in order to characterize the magnetic exchange at low temperature. Consistent with the spin reorientation that occurs below $140 \mathrm{~K}$, we do not observe a spin gap in this system above our experimental resolution. A Heisenberg model was fit to the spin wave data in order to characterize the long-range nature of the exchange. It was found that interactions up to sixth nearest neighbor are required to fully parameterize the spin waves. Surprisingly, the nearest-neighbor term is antiferromagnetic, and the realization of a ferromagnetic ground state relies on the more numerous ferromagnetic terms beyond nearest neighbor, suggesting that the ferromagnetic ground state arises as a consequence of the long-ranged interactions in the system.

The unusual magnetic properties of MnBi produce a material that is an attractive candidate as an alternative to rare-earth-containing ferromagnets in applications at room temperature and above ${ }^{1}$. Its high transition temperature $\left(\mathrm{T}_{C}=630 \mathrm{~K}\right)$ and strong magnetic anisotropy ${ }^{2 / 4}$ combined with large $\mathrm{Mn}$ moments $\left(3.50(2) \mu_{B}\right.$ at $300 \mathrm{~K}$, and $3.90(2) \mu_{B}$ at $5 \mathrm{~K}^{(5)}$ ) produce energy products up to $7.7 \mathrm{MGOe}^{4}$. Of particular interest for applications, MnBi has a magnetic coercivity that increases from $\approx 1 \mathrm{~T}$ at room temperature to $\approx 2.5 \mathrm{~T}$ at $550 \mathrm{~K}^{6-8}$. It also displays favorable magneto-optical properties such as a large Kerr effect, which may be useful as a magnetic storage medium in thin films $5[10$. For this reason, there is an ongoing, concerted effort to understand the growth conditions to optimize its properties for these applications 11 .

Despite the recent interest in $\mathrm{MnBi}$, the microscopic origin of its magnetic properties have not been completely determined. Above $140 \mathrm{~K}$, the ordered magnetic moments lie along the $c$-axis, but between $140 \mathrm{~K}$ and $90 \mathrm{~K}$ the spins undergo a continuous rotation away from the $c$-axis so that below $90 \mathrm{~K}$ the spins lie in the $a b$-plane $e^{4}$. This transition has been identified as a symmetry-lowering structural phase transition driven by magnetostriction ${ }^{5}$. It has been suggested that the Bi atoms play an important role in driving this spin-

\footnotetext{
a)Electronic mail: williamstj@ornl.gov
}

reorientation, as the $\mathrm{Bi}-\mathrm{Bi}$ exchange interactions have been proposed as an origin of the observed anisotropic thermal expansion ${ }^{5}$. Local spin density approximation (LSDA) calculations suggest that the spin reorientation occurs due to an inversion of the orbital momentum minimum on the $\mathrm{Bi}$ atom ${ }^{12}$, while a tight-binding atomicsphere-approximation (ASA) model predicts a small, antiparallel moment on the $\mathrm{Bi}$ atom, which undergoes a simultaneous spin reorientation ${ }^{4}$.

The loss of magnetic order above $630 \mathrm{~K}$ is not a typical magnetic transition, but occurs instead due to a decomposition of $\mathrm{MnBi}$ into $\mathrm{Mn}_{1.08} \mathrm{Bi}$ and elemental $\mathrm{Bi}$, both paramagnetic at this temperature. The product $\mathrm{Mn}_{1.08} \mathrm{Bi}$ has excess $\mathrm{Mn}$, where the excess $\mathrm{Mn}$ atoms occupy $8 \%$ of the interstitial vacancies present in $\mathrm{MnBi}^{\sqrt{6}}$. The relative proximity to the eutectic point $(535 \mathrm{~K})$ makes the synthesis difficult, which is why most prior work has been performed on samples that are $\mathrm{Mn}_{1.08} \mathrm{Bi}$ and/or polycrystalline ${ }^{3 / 418}$. Due to coupling of the magnetism with the structural decomposition, it has been found that application of a $10 \mathrm{~T}$ magnetic field can stabilize the MnBi phase up to $650 \mathrm{~K}^{10416}$. By cooling the melt in a magnetic field, MnBi crystallites can be obtained, as described elsewhere ${ }^{5|15| 17}$. Other isostructural Mn-based ferromagnets, such as MnSb ${ }^{18}$, cannot be synthesized in this manner and so they contain some degree of interstitial Mn. Thus, studying the interstitial-free $\mathrm{MnBi}$ presents the opportunity to understand the magnetism and electronic correlations in the most fundamen- 
tal form - coincidentally, the member of this family with the highest $\mathrm{T}_{C}$ and largest energy product ${ }^{4}$.

In this work, we use neutron scattering on a single crystal of $\mathrm{MnBi}$ that is free of interstitial $\mathrm{Mn}$ in order to measure the spin waves at low temperature. These measurements permit us to quantitatively determine the exchange interactions in this system, characterizing the degree of extended exchange that is present. Comparing this to other Mn-based binary magnets demonstrates the role that the long-ranged interactions play in the magnetic properties of this class of materials.

MnBi crystallizes in the hexagonal NiAs-type structure (space group $\mathrm{P}_{3} / m m c$ ), with the $\mathrm{Bi}$ atoms occupying half of the interstitial sites $3 \sqrt{4}$. For this study, single crystals of $\mathrm{MnBi}$ were grown from a flux utilizing excess $\mathrm{Bi}$, as described elsewhere ${ }^{5}$. The crystals have been characterized using powder and single-crystal x-ray diffraction measurements, whose refinements gave a nominal composition of $\mathrm{Mn}_{1.01(1)} \mathrm{Bi}$, where excess $\mathrm{Mn}$ atoms occupy interstitial sites ${ }^{5}$. This can be seen in Fig. 11. where the open circles denote the interstitial positions that can be partially filled by the excess Mn atoms.

For the neutron scattering measurements, a single crystal of mass $1.989 \mathrm{~g}$ and a mosaic of $0.41^{\circ}$ was rotated over a $90^{\circ}$ range in $1^{\circ}$ steps about a vertical axis, with the $\left[\begin{array}{lll}H & 0 & L\end{array}\right]$ plane horizontal. The measurements were performed on the Wide Angular-Range Chopper Spectrometer (ARCS) at the Spallation Neutron Source (SNS) 19 using incident neutron energies of $80 \mathrm{meV}$ and $150 \mathrm{meV}$, giving an energy resolution of 3.2 and $6 \mathrm{meV}$, respectively, at the elastic line. The sample was measured in a closed-cycle refrigerator at temperatures of $5 \mathrm{~K}$ and $200 \mathrm{~K}$.

The measured magnetic Bragg peaks at $5 \mathrm{~K}$ and $200 \mathrm{~K}$ are consistent with moments directed perpendicular and parallel to the $c$-axis, respectively. The $5 \mathrm{~K}$ data, which has reduced phonon scattering due to the Bose factor, was used to determine the magnetic exchanges in this system. The upper panels of Fig. 2 show representative slices through the spin wave spectrum measured on the ARCS spectrometer at $\mathrm{T}=5 \mathrm{~K}$, below the spin reorientation, so that the magnetic moments lie within the $a b$-plane. The spin waves were measured across multiple directions in reciprocal space, which allows the exchange interactions to be quantified. We see that the spin waves are well-defined, with no broad, inelastic features present in the data that would indicate the presence of significant quantities of interstitial Mn, as is observed for the case of $\mathrm{Mn}_{1.13} \mathrm{Sb}^{18}$ (shown in Fig. 2(e)). This is consistent with the refinement of diffraction data that measured 1(1)\% excess $\mathrm{Mn}$ in this sample and allows for a simpler determination of the exchange interactions since we do not have to account for the effects of interstitial Mn on the magnetic exchange.

To parametrize the spin wave dispersion, the data was fit to a Heisenberg model. The moment was assumed to be $3.90 \mu_{B}$ on the Mn atoms ${ }^{5}$, while no moment was placed on the $\mathrm{Bi}$ atoms. This is because the theoreti-

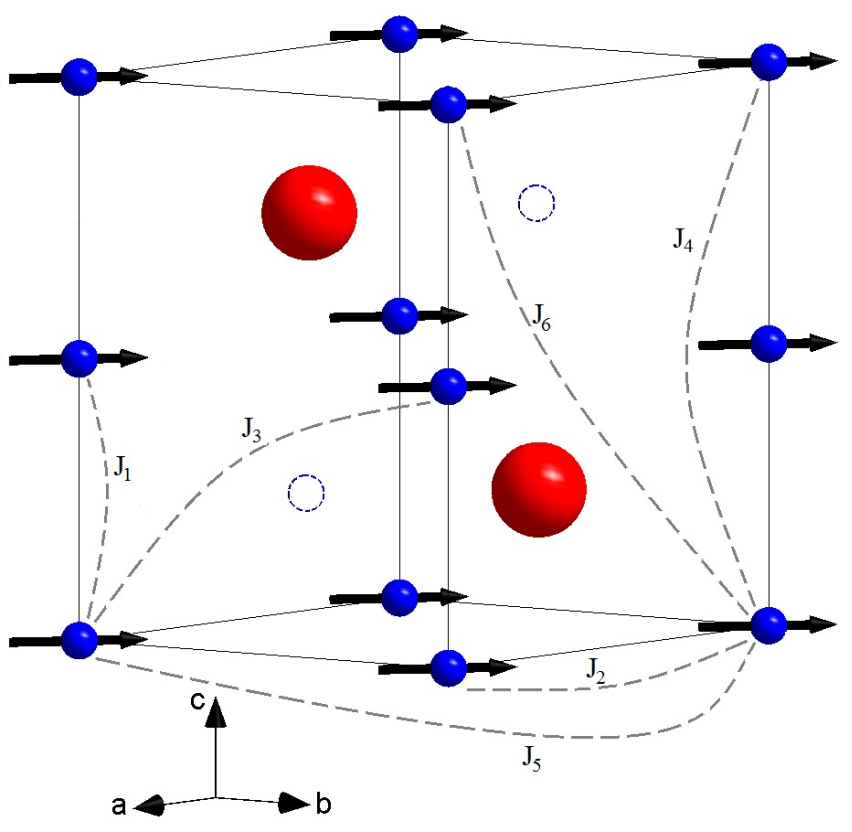

FIG. 1. (Color online) The crystal structure of MnBi. The Mn atoms (small, blue) occupy the $2 a$ position, while the $\mathrm{Bi}$ atoms (large, red) occupy the $2 c$ position. The other half of the interstitial positions (Wyckoff symbol $2 d$ ), shown as open circles, can be occupied by Mn impurities. Characterization measurements of the samples used suggested that 1(1) \% of the interstitial sites were so occupied by Mn atoms ${ }^{5}$. The spin arrangement shown is for the low-temperature range $(\mathrm{T}<90 \mathrm{~K})$. At all temperatures, $\mathrm{MnBi}$ is ferromagnetic, however above $\mathrm{T}=140 \mathrm{~K}$, the spins point atlong the $c$-axis, while from $\mathrm{T}=140 \mathrm{~K}$ to $90 \mathrm{~K}$, the spins rotate away from the $c$ axis such that below $90 \mathrm{~K}$ the spins lie entirely in the $a b$-plane. The exchange parameters $\left(J_{1}\right.$ through $\left.J_{6}\right)$ determined in this work are shown between the relevant $\mathrm{Mn}$ ions.

cal prediction for the size of the Bi moments is two orders of magnitude smaller than the Mn moments, and any moment on the $\mathrm{Bi}$ sites and accompanying $\mathrm{Bi}-\mathrm{Bi}$ exchange interactions would be too weak to be observed in these measurement\$12. In order to accurately reproduce the spin wave measurements, it was necessary to include interactions up to the $6^{\text {th }}$ nearest neighbor $\left(J_{6}\right)$, corresponding to a distance of $7.462 \AA$. These exchange interactions are shown schematically in Fig. 1. The need to include interactions up to $d=7.5 \AA$ indicates the significance of relatively long-ranged interactions, likely due to an itinerant nature of the magnetic exchange. Similarly, other isostructural Mn binary systems $\mathrm{MnP}^{20}$, $\mathrm{MnA}^{2122}$, and $\mathrm{MnSb}^{18 \mid 23}$ all have large Mn moments and all order ferromagnetically at, or near, ambient pressure. Despite the symmetry-lowering transition at $\mathrm{T}_{S R}=90 \mathrm{~K}$, the high-temperature space group (NiAs-type) was used for the spin-wave calculations as previous measurements of the symmetry-lowering distortion indicated that the magnitude of the distortion is very small, and thus unlikely to significantly influence the 


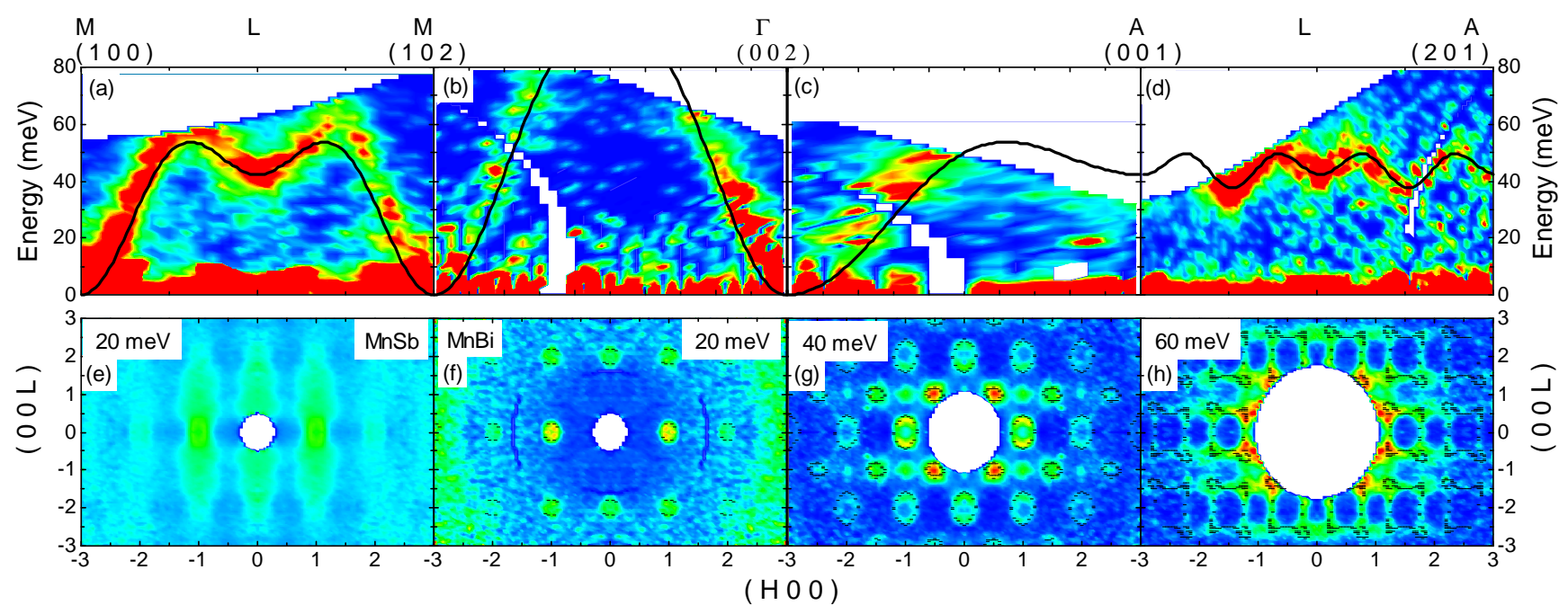

FIG. 2. (Color online) Spin wave measurements and fits of $\mathrm{MnBi}$ at $\mathrm{T}=5 \mathrm{~K}$. (a) to (d): Inelastic neutron measurements using $\mathrm{E}_{i}=150 \mathrm{meV}$ along different reciprocal space directions in the $\left(\begin{array}{lll}H & 0 & L\end{array}\right)$ scattering plane. The line is a fit to the model described in the text, with the parameters given in Table I Bottom row: Slices of the MnBi data in the $(H 0 L)$ scattering plane at $20 \mathrm{meV}(\mathrm{f}), 40 \mathrm{meV}(\mathrm{g})$ and $60 \mathrm{meV}(\mathrm{h})$, integrating the data $\pm 5 \mathrm{meV}$. The inelastic slices show the form factor dependence of the spin wave intensity, indicating their magnetic origin. (e) A slice of the related material $\mathrm{Mn}_{1.13} \mathrm{Sb}_{\mathrm{at}} 20 \mathrm{meV}$ (data taken from ${ }^{18}$ ). Compared to panel (f), we see that there is additional diffuse scattering along $L$ in $\mathrm{Mn}_{1.13} \mathrm{Sb}_{\text {, arising from }}$ the presence of interstitial Mn. The lack of similar elastic or inelastic signatures is consistent with the MnBi crystal studied being virtually free of interstitial $\mathrm{Mn}$.

magnetic excitation spectrum $[5$. For more details of the fitting procedure, see the Supplemental Informatione 24 .

The magnetic anisotropy present in this system necessitates a non-zero spin gap. However, the possible

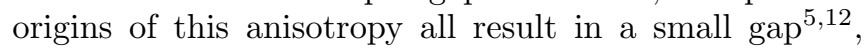
which agrees with the measurements of Fig 2, where any spin gap is less than the resolution of the data, approximately $6 \mathrm{meV}$. Measurements on $\mathrm{MnSb}$ at low temperatures likewise observed no gap, with a resolution of $0.5 \mathrm{meV}$. Moreover, the system undergoes a spin reorientation between $140 \mathrm{~K}$ and $90 \mathrm{~K}$ suggesting that the magnetic anisotropy is small and strongly temperaturedependent $t^{5}$. For these reasons, no spin gap term was included when fitting the data. The line through the data of Fig. 2 is the fit, showing that the dispersion was able to be accurately reproduced with the model that only included the Mn-Mn exchange terms given below, without any terms to account for Bi moments or a spin gap, though these may still be present. The lower panels of Fig. 2 show constant energy slices from the related material $\mathrm{Mn}_{1.13} \mathrm{Sb}$ at $20 \mathrm{meV}$, 18 , and from $\mathrm{MnBi}$ at $20 \mathrm{meV}$, $40 \mathrm{meV}$ and $60 \mathrm{meV}$. Comparing both $20 \mathrm{meV}$ slices, we see that there is additional scattering in $\mathrm{Mn}_{1.13} \mathrm{Sb}$ along the $L$-direction, arising from the presence of interstitial $\mathrm{Mn}$. The lack of that scattering in the MnBi data is consistent with the diffraction measurements that have only a small amount of interstitial $\mathrm{Mn}^{\underline{5}}$.

The values of the exchange interactions are shown in Table I. The effective nearest-neighbor interaction is strongly antiferromagnetic, while the rest of the terms are

\begin{tabular}{|c|c|l|c|c|}
\hline Exchange & No. Neighbors & Vector & Distance & Value $(\mathrm{meV})$ \\
\hline$J_{1}$ & 2 & $c / 2$ & $3.055 \AA$ & $4.70(17)$ \\
$J_{2}$ & 6 & $a$ & $4.283 \AA$ & $-0.61(10)$ \\
$J_{3}$ & 12 & $a+c / 2$ & $5.261 \AA$ & $-1.73(3)$ \\
$J_{4}$ & 2 & $c$ & $6.110 \AA$ & $-0.12(18)$ \\
$J_{5}$ & 6 & $a+b$ & $7.418 \AA$ & $-1.29(8)$ \\
$J_{6}$ & 12 & $a+c$ & $7.462 \AA$ & $-0.63(3)$ \\
\hline
\end{tabular}

TABLE I. The values for the exchange constants obtained from fitting the data in Fig. 2 where positive values denote antiferromagnetic exchange. In order to model the data, it was necessary to include up to $6^{\text {th }}$ nearest-neighbor interactions due to the extended nature of the exchange.

smaller and ferromagnetic. However, due to the larger number of neighbors at greater distances, the ferromagnetic terms more than compensate for the $J_{1}$ term, leading to a ferromagnetic ground state. The antiferromagnetic $J_{1}$ term is somewhat unexpected, due to the large Mn-Mn distance ${ }^{25 \mid 26}$, but a fit that forced $J_{1}$ to be ferromagnetic did not reproduce the data. As a further consistency check on the nature of the magnetic interactions, we have performed first principles calculations of the effective Heisenberg exchange parameters using the generalized gradient approximation $(\mathrm{GGA})^{27}$ in the planewave all-electron density functional theory code WIEN2K $\mathrm{K}^{28}$ (see the Supplemental Information) ${ }^{24}$. In agreement with the experiment, $J_{1}$ is found to be large and antiferromagnetic, but the ground state is ferromagnetic due to mod- 
erate ferromagnetic values for $J_{3}$ and $J_{5}$. The remaining exchange constants are all small, as was found in the fits to the experimental data. The calculations find that the antiferromagnetic state lies some $135 \mathrm{meV}$ per Mn higher than the ferromagnetic ground state, leading to a mean-field estimated Curie temperature of $522 \mathrm{~K}$, in reasonable agreement with experiment. While the nearestneighbor exchange term being antiferromagnetic is unexpected, both the calculations and the experimental data conclude that the long-ranged nature of the Mn exchange give rise to ferromagnetism. This competition between antiferromagnetic local exchange and the ferromagnetic itinerant exchange component may also be responsible for the reduction of the moment to $3.90 \mu_{B}$ from the nominal moment of $g S=4 \mu_{B}$. . However, the long-range character in MnBi clearly dominates, especially when compared to isostructural and isoelectronic Mn binary systems (see Table II].

\begin{tabular}{|l|c|c|c|}
\hline Compound & $T_{\text {Curie }}(\mathrm{K})$ & Moment $\left(\mu_{B}\right)$ & NN Mn Dist. $(\AA)$ \\
\hline $\mathrm{MnB}$ & 630 & 3.90 & 3.055 \\
$\mathrm{MnSb}$ & 587 & 3.55 & 2.895 \\
$\mathrm{MnAs}^{22 \mid 31}$ & 318 & 3.20 & 2.852 \\
$\mathrm{MnP}^{20 \mid 32}$ & 292 & 1.33 & 2.743 \\
\hline
\end{tabular}

TABLE II. Magnetic properties of various $\operatorname{Mn} T(T=\mathrm{Bi}, \mathrm{Sb}$, As, P) materials. These compounds are found to be ferromagnetic, where the distance between the $\mathrm{Mn}$ ions increases with increasing size of the $T$ ion. This suggests that there is a systematic increase in the role of the extended exchange, which results in higher moments and transition temperatures for larger $T$ atoms in the $\mathrm{Mn} T$ series. This agrees well with the results of the inelastic neutron scattering on $\mathrm{MnBi}$. ${ }^{\star} \mathrm{The}$ compound $\mathrm{MnP}$ is orthorhombic, a slight distortion of the NiAs-type structure of the other compounds listed here. This leads to a helical magnetic state below $50 \mathrm{~K}$, however the magnetic structure between $50 \mathrm{~K}$ and $\mathrm{T}_{C}=292 \mathrm{~K}$ is the same as for the other $\operatorname{Mn} T$ compounds.

Of these materials, MnBi has the highest Curie temperature and largest ordered moment. This is likely due to the larger inter-Mn distance, which reduces the nearest-neighbor exchange, and places increased emphasis on the higher-order exchange terms. Therefore we expect similar spin wave measurements of these compounds to demonstrate a systematic shift from long-ranged to more local interactions. These measurements are greatly complicated by the inclusion of interstitial $\mathrm{Mn}^{18}$, and it also remains an open question to what degree the interstitial ions influence the moment and exchange pathways in these various systems 30 . However, the systematic changes through the Mn binary ferromagnets are likely controlled by the varying importance of the long-range interactions.

In conclusion, we have observed well-defined spin waves in $\mathrm{MnBi}$. In order to characterize the spin waves, a Heisenberg model with six exchange constants has been fit to the data. The need to include terms out to $d=7.5 \AA$ suggests that the Mn-Mn exchange is itin- erant, and the data taken here shows that any gap in this material is less than $6 \mathrm{meV}$. The resulting fits to the data have shown that the nearest-neighbor term is antiferromagnetic, while all of the higher-order terms are ferromagnetic. This quantitative determination of the exchange parameters shows that the long-range interactions are the determining factor in the ferromagnetic ordering, a trend that can explain the reduction in ordered moment and Curie temperature in isostructural and isoelectronic Mn binary systems.

The strength and sign of the exchange constants in $\mathrm{MnBi}$ should form a basis for comparing to nonstoichiometric $\mathrm{Mn}_{1.08} \mathrm{Bi}$, as well as other $\mathrm{Mn}$ binary ferromagnets: $\mathrm{MnAs}, \mathrm{MnP}$ and $\mathrm{MnSb}$, particularly in understanding the role of interstitial $\mathrm{Mn}$ on the magnetic properties. Finally, the results obtained here shed light on the role of extended exchange in determining the essential characteristics of $\mathrm{MnBi}$ for use in permanent magnet applications.

We acknowledge instrument support from D.A. Abernathy and J. Niedziela. This research at ORNL's Spallation Neutron Source was sponsored by the Scientific User Facilities Division, Office of Basic Energy Sciences, US Department of Energy. T.J.W. acknowledges support from the Wigner Fellowship program at Oak Ridge National Laboratory. M.A.M. acknowledges support from U.S. Department of Energy, Office of Energy Efficiency and Renewable Energy, Vehicle Technologies Office, Propulsion Materials Program. B.C.S. and D.S.P. were supported by the Critical Materials Institute, an Energy Innovation Hub, funded by the U. S. Department of Energy, Office of Energy Efficiency and Renewable Energy, Advanced Manufacturing Office. R.S.F. was supported by the Department of Energy, Office of Science, Basic Energy Sciences, Materials Sciences and Engineering Division.

${ }^{1}$ N. Poudyal and J.P. Liu. J. Phys. D: Appl. Phys. 46, 043001 (2013).

${ }^{2}$ F. Heusler. Z. Angew. Chem. 17, 260 (1904).

${ }^{3}$ N. Poudyal and J.P. Liu. J. Phys. D: Appl. Phys. 46, 043001 (2013).

${ }^{4}$ J.B. Yang, W.B. Yelon, W.J. James, Q. Cai, S. Roy and N. Ali. J. Appl. Phys. 2002, 7866 (2002).

${ }^{5}$ M.A McGuire, H. Cao, B.C. Chakoumakos and B.C. Sales. Phys. Rev. B. 90, 174425 (2014).

${ }^{6}$ T. Chen and W.E. Stutius. IEEE Trans. Mag. 10, 581 (1974).

${ }^{7}$ X. Guo, X. Chen, Z. Altounian and J.O. Ström-Olsen. Phys. Rev. B. 46, 14578 (1992).

${ }^{8}$ J.B. Yang, Y.B. Yang, X.G. Chen, X. B. Ma, J.Z. Han, Y.C. Yang, S. Guo, A.R. Yan, Q.Z. Huang, M.M. Wu and D.F. Chen. Appl. Phys. Lett. 99, 082505 (2011).

${ }^{9}$ H.J. Williams, R.C. Sherwood, F.G. Foster and E.M. Kelley. J. Appl. Phys. 28, 1181 (1957).

${ }^{10}$ D.J. Sellmyer, R.D. Kirby, J. Chen, K.W. Wierman, J.X. Shen, Y. Liu, B.W. Robertson and S.S. Jaswal. J. Phys. Chem. Solids. 56, 1549 (1995).

${ }^{11}$ J. Cui, J.-P. Choi, E. Polikarpov, M.E. Bowden, W. Xie, G. Li, Z. Nie, N. Zarkevich, M.J. Kramer and D. Johnson. Acta Mater. 79, 374 (2014).

${ }^{12}$ V.P. Antropov, V.N. Antonov, L.V. Bekenov, A. Kutepov and G. Kotliar. Phys. Rev. B. 90, 054404 (2014). 
${ }^{13}$ T. Chen. J. Appl. Phys. 45, 22358 (1973).

${ }^{14}$ E. Adams. Rev. Mod. Phys. 25, 306 (1952).

${ }^{15}$ Y. Liu, J. Zhang, S. Cao, X. Zhang, G. Jia, Z. Ren, X. Li, C. Ling and K. Deng. Phys. Rev. B. 72, 214410 (2005).

${ }^{16} \mathrm{~T}$. Onogi, K. Koyama and K. Watanabe. Journal of the Japan Institute for Metals. 71, 489 (2007).

${ }^{17}$ H. Morikawa, K. Sassa and S. Asai. Materials Transitions JIM. 39, 814 (1998).

${ }^{18}$ A.E. Taylor, T.Berlijn, S.E. Hahn, A.F. May, T.J. Williams, L. Poudel, S. Calder, R.S. Fishman, M.B. Stone, A.A Aczel, H.B. Cao, M.D. Lumsden and A.D. Christianson. Phys. Rev. B. 91, 224418 (2015).

${ }^{19}$ D.L. Abernathy, M.B. Stone, M.J. Loguillo, M.S. Lucas, O. Delaire, X. Tang, J.Y.Y. Lin and B. Fultz. Rev. Sci. Instr. 83, 015114 (2012).

${ }^{20}$ H. Obara, Y. Endoh, Y. Ishikawa and T. Komatsubara. J. Phys. Soc. Japan. 49, 928 (1980).

${ }^{21}$ N. Menyuk, J.A. Kafalas, K. Dwight and J.B. Goodenough. Phys. Rev. 177, 942 (1969).

${ }^{22}$ S. Gama, A.A. Coelho, A. de Campos, A.M.G. Carvalho, F.C.G. Gandra, P.J. von Ranke and N.A. de Oliviera. Phys. Rev. Lett. 93, $237202(2004)$

${ }^{23}$ C. Guillard. Ann. de Phys. Paris. 4, 671 (1949).

${ }^{24}$ See $U R L$ for a detailed description of the fitting procedure and first principles calculations used in this work.

${ }^{25}$ R. Forrer. Ann. de Phys. 7, 605 (1952).

${ }^{26}$ V. Seshu Bai and T. Bajasekharan. J. Mag. Mag. Mat. 42, 198 (1984).

${ }^{27}$ J.P. Perdew, K. Burke, and M. Ernzerhof. Phys. Rev. Lett. 77 , 3865 (1996).

${ }^{28}$ P. Blaha, K, Schwarz, G. Madsen, D, Kvasnicka and J. Luitz, WIEN2k, An Augmented Plane Wave + Local Orbitals Program for Calculating Crystal Properties. (K. Schwarz, Tech. Univ. Wien, Austria, 2001).

${ }^{29}$ K. Elankumaran, G. Markandeyulu and K.V.S. Rama Rao. J. Phys. Soc. Japan. 61, 1979 (1992).

${ }^{30}$ P. Radhakrishna and J.W. Cable. Phys. Rev. B. 54, 11940 (1996).

${ }^{31}$ M. Yuzuri and M. Yamada. J. Phys. Soc. Japan. 15, 1845 (1960).

${ }^{32}$ A. Continenza, S. Picozzi, W.T. Geng and A.J. Freeman. Phys. Rev. B. 64, 085204 (2001). 


\section{Supplemental Information for Williams et al. Extended Magnetic Exchange Interactions in the High-Temperature Ferromagnet MnBi}

\section{DATA FITTING PROCEDURE}

The data was fit to a Heisenberg model given by:

$$
H=J_{n} \sum_{<i, j>} \mathbf{S}_{\mathbf{i}} \cdot \mathbf{S}_{\mathbf{j}}
$$

where $J_{n}$ is the exchange interaction between the $n$-th nearest neighbors $S_{i}$ and $S_{j}$, taking the convention that positive values of $J_{n}$ denote antiferromagnetic exchange. In order to parametrize the data we needed to include nearest-neighbors out to $d=7.5 \AA$, which requires exchange interactions up to $J_{6}$. Fits using fewer exchange constants did not reproduce the data, particularly along ( $\left.\begin{array}{lll}H & 0 & 1\end{array}\right)$ (shown in Fig. S1(d)) where the $6^{\text {th }}$ nearest neighbor term contributes an additional sinusoidal term.

Constant- $Q$ cuts were made through the data and the cuts were fit to a Gaussian to extract the peak energy for each cut. This array of 25 points were taken from the following direc-

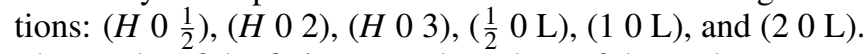
The results of the fitting gave the values of the exchange constants listed in Table I of the Letter. The errors were calculated by the method described in ${ }^{1}$. Using these values, the data was simulated to produce plots of $S(Q, \omega)$ shown in Fig.S1, below.

No resolution corrections were included in the fits, but the plots below have been convoluted with a two-dimensional Gaussian function to approximate the energy- and $Q$ resolution of the instrument. The magnetic form factor is also included in these plots.

\section{FIRST PRINCIPLES CALCULATIONS}

The principal question theory should answer regarding $\mathrm{MnBi}$ is the following: how is it possible that in a magnetic material containing Manganese - which usually prefers antiferromagnetic behavior - ferromagnetic behavior arises, and most crucially, how does ferromagnetic behavior arise despite the nearest neighbor Mn-Mn coupling being antiferromagnetic?

To understand the magnetic behavior of $\mathrm{MnBi}$, we have performed first principles calculations of the effective Heisen- berg exchange parameters $J_{i j}$. using the generalized gradient approximation $(\mathrm{GGA})^{2}$ in the planewave all-electron density functional theory code WIEN $2 \mathrm{~K}^{3}$. One recalls that in such a Heisenberg model, usually applicable to systems such as $\mathrm{MnBi}$ where the magnetic element carries a sizable local moment, the system energy can be taken (relative to a zero of energy) as

$$
E=\sum_{<i, j>} J_{i, j} \mathbf{S}_{\mathbf{i}} \cdot \mathbf{S}_{\mathbf{j}}
$$

where the $\langle i, j\rangle$ represent first, second, etc. nearest neighbors and it is presumed that a single $\mathbf{J}_{i, j}$ is applicable to all atom neighbor pairs $\langle i, j\rangle$ of equal separation. Here the $\mathbf{S}$ represent the Pauli spin-operators. Note that while in Eq. 1 the magnitude of the spin (i.e. $S(S+1)$ ) enters, in the following paragraphs we treat $\mathrm{S}(\mathrm{S}+1)$ as unity; to extract actual $J_{i, j}$ values one should divide by $3.77 *(3.77+1) \sim 18$ (since the calculated Mn spin moment is approximately $3.77 \mu_{B}$.)

The results of the calculations are as follows. Firstly, in agreement with experiment, of all the configurations modeled the ferromagnetic case has the lowest energy. The simplest antiferromagnetic case, in which the Mn-Mn nearest neighbors along the c-axis are anti-aligned, lies some $135 \mathrm{meV}(\sim \Delta E)$ per Mn higher, leading to a mean-field Curie point estimate $T_{c}=\Delta E / 3=522 \mathrm{~K}$, in reasonable agreement with the experimental value of $630 \mathrm{~K}$. Also in agreement with experiment, the nearest-neighbor $J_{1}=4.22 \mathrm{meV}$, indicating a tendency towards antiferromagnetic alignment. The second nearestneighbor (the in-plane nearest-neighbor), $\mathrm{J}_{2}$ at $1.74 \mathrm{meV}$ is also positive. However, $\mathrm{J}_{3}-\mathrm{J}_{6}$ are all negative, and in particu$\operatorname{lar} \mathrm{J}_{3}$ is $-6.33 \mathrm{meV}$ and $\mathrm{J}_{5}$ is $-8.33 \mathrm{meV}$. Given that these last two J's comprise 12 and 6 neighbor pairs, respectively, the combination of these exchange constants provides an energy incentive of over $100 \mathrm{meV}$ per $\mathrm{Mn}$ for ferromagnetic alignment, which in the calculations is the "driving force" for ferromagnetism in $\mathrm{MnBi}$. The NiAs hexagonal structure, with its large number of Mn-Mn distant neighbors and relatively small (2) number of nearest neighbors is clearly of great importance for the ferromagnetism.
1 P.R. Bevington and D.K. Robinson, Data Reduction and Error Analysis for the Physical Sciences. 3rd. Ed. (McGraw-Hill, New York, New York, USA, 2003).

2 J.P. Perdew, K. Burke, and M. Ernzerhof, Generalized gradient ap- proximation made simple, Phys. Rev. Lett. 77 , 3865 (1996).

3 P. Blaha, K, Schwarz, G. Madsen, D, Kvasnicka and J. Luitz, WIEN2k, An Augmented Plane Wave + Local Orbitals Program for Calculating Crystal Properties (K. Schwarz, Tech. Univ. Wien, Austria, 2001). 


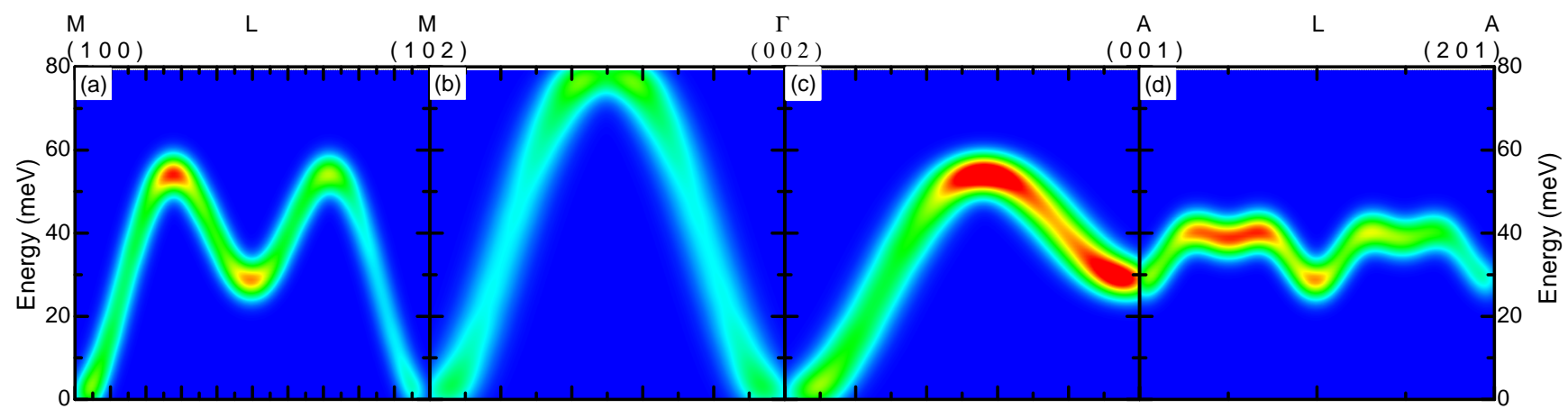

FIG. S1. (Color online) Simulations of the data based on the exchange constants found in the fitting procedure described above, shown along the same directions in reciprocal space as the data in Fig. 2(a) to (d) of the Letter. The instrumental resolution has been been included by convolving the fit with a two-dimensional Gaussian, and the magnetic form factor has also been applied. 